\title{
YOGA FOR BLISSFUL AGEING
}

\section{Mahesh Pandurang Patil ${ }^{* 1}$, Soumya Shrinivas Bhujle ${ }^{2}$, Chintamani D. Patil ${ }^{3}$}

1. Asst. Professor, Dept. of Rachana Sharir, GAMRC, Shiroda, Goa.

2. BAMS, Panaji,Goa. Email Id: soumyabhujle4@gmail.com Phone no. 7507378732

3. PG Scholar, Panchakarma Dept. LKRAMC, Gadhinglaj, Maharashtra.

Email Id - chintspatil86@ gmail.com Phone no. 8552888796.

*Corresponding author: Email: drmaheshppatil@ gmail.com; Phone no. 9923638626

\begin{abstract}
:-
Growing or ageing is the natural process of getting old. Populations around the world are rapidly ageing. There are many factors that play important role in accelerating this process, leading to premature ageing. The causative factors for accelerating the process include diet, lifestyle smoking alcohol consumption, etc. Today's lifestyl demands ignorance towards the mind health leading to stress, which is one of the very important factors that affects the healthy ageing in the recent era. While premature ageing is setting many alarming signs to the human health; Yoga here plays an important role offering many solutions, both at the ground of body as well as mind. This paper explains about the contribution of yoga for healthy ageing and preventing the factors for premature ageing and helps in blissful ageing.
\end{abstract}

KEY WORDS :- Yoga, Ageing, Stress

\section{INTRODUCTION :-}

Populations around the world are rapidly ageing. Today, most people are living longer and a significant proportion of the world's population are older people. As per WHO by 2050, the world's population of people over the age of 60 will double from $12 \%$ to $22 \%$. Ageing healthily is what we all aspire to. Healthy Ageing is about maintaining the functional ability that allows you to do the things you value. This means preserving both your physical and mental capacity as you age. The major concern in the current era is the "Premature ageing" and not the natural ggeing. There are various causes of premature ageing like lifestyle, Excessive and Restrictive Dieting, smoking, alcohol consumption, lack of sleep, environmental factors etc. But the most important and prominent of which is stress, which is often neglected.

\section{METHODS :-}

The literature search was performed using the following electronic database: PubMed, Google Scholar, Cochrane Library, Science Direct, WHO and Web of Science. The search terms used contained: "stress", "ageing" AND "yoga." Articles containing research done on effects of stress, premature ageing, prevention of stress with yoga, asanas, meditation etc. were reviewed, screened and used for deriving conclusion in this article.

\section{Stress as cause in premature ageing :-}

Chronic psychological stress is a risk factor for multiple diseases of aging. Accelerated cellular aging as indexed by 
short telomere length has emerged as a potential common biological mechanism linking various forms of psychological stress and diseases of aging. Stress appraisals determine the degree and type of biological stress responses and altered stress appraisals may be a common psychological mechanism linking psychological stress and diseases of aging.

Stress can affect our body, our thoughts and our behavior. Stress that's left unchecked can contributes to many health problems, such as high blood pressure, heart disease, obesity and diabetes.

\section{Common effects of stress on our body and mind}

- Headache

- Muscle tension or pain

- Chest pain

- Fatigue

- Stomach upset

- Sleep problems

- Anxiety\& Restlessness

- Irritability or anger

- Sadness or depression

Chronic stress can also plays an important factor for accelerating ageing process.

A 2012 study published in PLoS ONE journal, found that work related exhaustion have a harmful effect on critical DNA in cells. Telomeres were found to be the shortest in such cases. ${ }^{1}$

A study shows that even the anticipation of stress accelerates the ageing process.UC San Francisco research found that mere anticipation also led to ageing at the cellular level. $^{2}$

UC Berkleys scientists found that the pattern of gene activation and de activation that occurs as the brain ages, seemed to progress quickly due to stress. $^{3}$
In addition to tachycardia and HTN, increased levels of adrenaline leads to temporary .vision and hearing loss and reduce their vitality on a permanent basis. ${ }^{4,5,6}$

Sustained high levels of cortisol's result in breaking up of collagen and elastin hence leading to early wrinkling of skin hence early ageing. ${ }^{7}$

Over the past several years, it has become increasingly clear that alterations in telomere integrity can directly impact human health. At the molecular level, telomeres are long sequences of noncoding, six-nucleotide-long tandem repeat of "TTAGGG" DNA bases that are located at the terminal ends of all vertebrate chromosomes, including those of humans. When the telomere length becomes critically short, it can no longer protect the cell's DNA, leaving the cell at risk for serious damage. Critical telomere shortening leads to telomere and chromosomal instability which promotes genomic rearrangements that impinge upon disease-relevant pathways.Under physiological conditions, such unstable telomere has been associated with diseases linked with aging and stress exposure, including diabetes mellitus, obesity, heart disease, chronic obstructive pulmonary disease, asthma, cancer, as well as psychiatric illnesses, such as depression, anxiety, posttraumatic stress disorder, bipolar disorder, and schizophrenia. Stress is one the factor which significantly increase the rate of telomere shortening. ${ }^{8,9,10}$

\section{Importance of yoga for stress prevention and blissful ageing :-}

A number of interventions have been studied to determine their influences on stress control and telomere stability as indices for promoting health and longevity. Among them, yoga seems to provide comprehensive benefits by delaying or reversing accelerated 
pathological manifestations of diseases. Yoga is an ancient mind-body practice which includes meditation, breathing practices, asanas, philosophy, cleansing practices, and deep relaxation. It is believed that regular practice of yoga brings about a decrease in stress levels and improved antioxidant status by establishing natural harmony and functional balance between various organ systems, leading to better health and a feeling of well-being. In spite of the positive effects of yoga which have been demonstrated through disease-specific outcomes, basic understanding of the mechanism in terms of modern sciences is not very clear. However, there is evolving evidence that regular practice of asana, pranayama, and meditation help to combat stress and reduce stress related problem. ${ }^{11}$

When we are in a constant state of stress, our minds are tense, our bodies are tense and our sympathetic nervous system is heightened. Specific yoga poses can induce the relaxation response in the body Calming and restorative poses along wit controlled breathing activate the parasympathetic nervous system, bringing the mind and body to a calm and relaxed state.

\section{Some Asanas that help in stress management :-}

\section{Padmasana :}

This pose is commonly used during meditation and helps to increase the selfawareness levels. The pose helps you to calm down and soothes your mind.

2. Tadasana:

It helps you improve your concentration levels by increasing your focus level

3. Adho Mukha Svanasana:

Along with energizing the body and offering relief from indigestion problems, the pose is known to relax your body.
Thus, it is an ideal asana for obtaining relief from stress.

\section{Shavasana:}

Relax yourself completely with this pose. If you do not have time for any other yoga asanas, you can practice this. It brings your breathing to normal levels, and thus, helps to soothe down stress levels.

The study led by Tolahunase et al. ${ }^{12}$ which evaluated the impact of cellular aging in apparently healthy individuals found telomerase activity to be significantly increased $(P<0.05)$ in the yoga intervention group. The intervention included a set of a sanas, pranayama, and meditation for approximately $90 \mathrm{~min}$ for 12 weeks. The group also found similar results in another study involving a 31year-old man with class I obesity (body mass index, $29.5 \mathrm{~kg} / \mathrm{m}^{2}$ ). The patient was asked to perform a series of asanas, pranayama, and meditation for approximately $1 \mathrm{~h}$ for 3 months. After the interventional period, the activity of telomerase increased, and a sustained reduction in oxidative stress markers, such as ROS and 8-oxoG, as compared to baseline levels was seen. ${ }^{13}$

\section{Pranayama :}

With our busy life schedules, we often ignore our breathing. It tends to be fast and shallow. Central to asanas and pranayama is diaphragmatic breathing which is the act of breathing deeply into the lungs by flexing the diaphragm rather than the rib cage. The basic mode of respiration used in yoga practice is slow, smooth breathing using the diaphragm rather than respiratory muscle of chest. Asanas involving diaphragmatic breathing can increase alveolar gas exchange and ventilatory distribution and improve submaximal and maximal oxygen consumption.Hence, pranayama can alter the perceptions and mental responses to both external and internal stimuli, slow down reactivity, and reduce ROS production. ${ }^{13,14}$ 
Some Pranayamas that help in stress management:

\section{AnulomVilom Pranayama :}

It induces calmness of mind by regulating the flow of prana in the body. The whole body is nourished with an extra supply of pure oxygen, and the carbon dioxide is more efficiently eliminated. Found to be good to increase concentration, to get sound sleep.

\section{Sheetali Pranayama :}

It helps to cool the body and affects the important brain centers associated with biological drives and temperature regulation. It reduces mental and emotional excitation, and encourages the free flow of prana throughout the body. It induces muscular relaxation, mental tranquility.

\section{Bhramari Pranayama :}

Bhramari relieves stress and cerebral tension, so helps in alleviating anger, anxiety and insomnia, increasing the healing capacity of the body. It strengthens and improves the voice. Bhramari induces a meditative state by harmonizing the mind and directing the awareness inward. The vibration of the humming sound creates a soothing effect on the mind and nervous system

A 2015 randomized controlled study published in the journal Cancer, found yoga and meditation maintained the telomere length in pt. of breast cancer.Researchers at the University of Calgary Alberta, Canada administered yoga to 88 breast CA survivors and it was found that the practice maintained their telomere length. ${ }^{15}$

Researchers at Harvard Medical School obtained blood samples from 15 meditators \& 22 non meditators. Here it was studied that the former had longer telomeres than the latter. ${ }^{16}$
In a study at Thomas Jefferson Medical College in Philadelphia and Yoga Research Society, 16 yogis had participated where it was noticed that with sarvangaasan,shalbhaasan, vrikshaasan and halaasan, there was significant drop in the levels of cortisol. ${ }^{17,18}$

\section{DISCUSSION :-}

The present systemic review indicates a positive association between stress and cellular aging and its prevention with intervention programs involving yoga and meditation.

Since asanas involve slow and steady muscle stretch, it allows for effortless, easy, and comfortable maintenance of the posture, allowing various muscle and joints to stretch smoothly without any resistance. This static but passive stretching of the muscles and ligament affords sufficient time to stimulate the circulation around various tissues and rgans. This also causes effective and easy removal of waste products of the metabolism from the body. Hence, asanas itself reduce oxidative stress and prevent toxic buildup of metabolites in the body, which ultimately reduces molecular damage to cells.

Currently, treatment for anxiety and depression involves mostly psychological and pharmacological interventions; however, mind-body interventions are becoming increasingly popular as a means to reduce stress. Yoga, a form of mindbody exercise, has become an increasingly widespread therapy used to maintain wellness, and alleviate a range of health problems and ailments.

The results of this review highlight the positive effects of yoga intervention on prevention of causes leading to premature ageing and helps in healthy or blissful ageing.A major limitation of this review is that only few clinical trials have been found in the literature.More rigorous 
randomized controlled trials of healthy controls and specific disease states are needed.

\section{CONCLUSION :-}

A growing body of research evidence supports the belief that certain yoga techniques may improve physical and mental health through down-regulation of the hypothalamic-pituitary-adrenal (HPA) axis and the sympathetic nervous system (SNS). The HPA axis and SNS are triggered as a response to a physical or psychological demand (stressor), leading to a cascade of physiologic, behavioral, and psychological effects, primarily as a result of the release of cortisol and catecholamines (epinephrine and norepinephrine). Over time, the constant state of hypervigilance resulting from repeated firing of the HPA axis and SNS can lead to dysregulation of the system, and ultimately diseases such as obesity, diabetes, autoimmune disorders depression, substance abuse, and cardiovascular disease. . Many studie have found that including yoga in daily routine, can minimize stress and increase productivity. Yoga offers an effective method of managing and reducing stress, anxiety, and depression, and numerous studies demonstrate the efficacy of yoga on stress related disorders and its effect on body.

\section{REFERENCES :-}

1. Work-Related Exhaustion and Telomere Length: A Population-Based Study. KirsiAhola, IlariSirén, Mika Kivimäki, SamuliRipatti, ArpoAromaa, JoukoLönnqvist, IirisHovatta

Published: July 11, 2012https://doi.org/10.1371/journal.po ne. 0040186

2. Stress appraisals and cellular aging: A key role for anticipatory threat in the relationship between psychological stress and telomere
lengthAoifeO'DonovanaA.

JanetTomiyamabcJueLindEliPuterman

aNancy

E.AdleraMargaretKemenyaOwen

M.WolkowitzaElizabeth

H.BlackburndElissaS.Epelahttps://doi. org/10.1016/j.bbi.2012.01.007

3. Accelerated aging-related transcriptome changes in the female prefrontal cortex

Yuan Yuan Yi-Ping Phoebe Chen Jerome Boyd-Kirkup Philipp Khaitovich Mehmet Somel

First published: 11 July 2012 https://doi.org/10.1111/j.1474-

9726.2012.00859.

4. Immediate effect of epinephrine on aqueous formation in the normal human eye as measured by fluorophotometry.D J Townsend; R F Brubaker

Investigative Ophthalmology \& Visual Science March 1980, Vol.19, 256-266. doi:

Load Independence of the Instantaneous Pressure-Volume Ratio of the Canine Left Ventricle and Effects of Epinephrine and Heart Rate on the Ratio. HIROYUKI SUGA , KIICHI SAGAWA, and ARTIN A. SHOUKAS

Originally published2 Apr 2018Circulation Research. 2018;32:314-322

6. https://jonbarron.org/article/endocrinesystem-adrenal-glands

7. Stress and depression-induced immune dysfunction: Implicationsfor the development and progression of canceREDNA MARIA VISSOCI REICHE1, HELENA KAMINAMI MORIMOTO1,\&SANDRA ODEBRECHT VARGAS NUNES https://www.researchgate.net/publicati on/7369473_Stress_and_depressioninduced_immune_dysfunction_Implica tions_for_the_development_and_progr ession_of_cancer [accessed Sep 07 2018]. 
8. Moyzis RK, Buckingham JM, Cram LS, Dani M, Deaven LL, Jones MD, et al. ProcNatlAcadSci U S A 1988;85:6622-6

9. Reddel RR. Alternative lengthening of telomeres, telomerase, and cancer. Cancer Lett 2003;194:155-62.

10. Telomerase activity in human germline and embryonic tissues and cells. Wright WE, Piatyszek MA, Rainey WE, Byrd W, Shay JW. Dev Genet 1996;18:1739.

11. Implication of asana, pranayama and meditation on telomere stability, Mrithunjay Rathore, Jessy Abraham, http://www.ijoy.org.in; year 2018; volume 11 ; issue- 3 ;

12. Impact of yoga and meditation on cellular aging in apparently healthy individuals: A prospective, open-label single-arm exploratory studyTolahunase M, Sagar R, Dada R..Oxid Med Cell Longev 2017;2017:7928981

13. Telomerase activity and cellular agin might be positively modified by yoga-based lifestyle interventioñ. Kumar SB, Yadav R, Yadav RK, Tolahunase M, Dada R.. J Altern Complement Med 2015;21:370-2.
14. Yoga as Medicine: The Yogic Prescriptions for Health and Living. McCall T. 1st ed. New York: Bantam Publishers; 2007.

15. Mindfulness-based cancer recovery and supportive-expressive therapy maintain telomere length relative to controls in distressed breast cancer survivors.Carlson LE1, Beattie TL, Giese-Davis J, Faris P, Tamagawa R, Fick LJ, Degelman ES, Speca M. Cancer. 2015 Feb 1;121(3):476-84. doi: 10.1002/cncr.29063. Epub 2014 Nov

16. Loving-Kindness Meditation practice associated with longer telomeres in women.Hoge EA1, Chen MM, Orr E, Metcalf CA, Fischer LE, Pollack MH, De Vivo I, Simon NM. Brain Behav Immun. 2013 Aug;32:159-63. doi: 10.1016/j.bbi.2013.04.005. Epub 2013 Apr 19

17. Yoga is shown to lower levels of stress hormones, June 30, 2003|Dianne Partie Lange,

http://articles.latimes.com/2003/jun/30/ health/he-capsules30.2

18. Destress with Yoga. LINDA KNITTELAUG 28, 2007, https://www.yogajournal.com/health/b eginner-s-bliss.

Cite article:

YOGA FOR BLISSFUL AGEING

Mahesh Pandurang Patil, Soumya Shrinivas Bhujle, Chintamani D. Patil

Ayurlog: National Journal of Research in Ayurved Science- 2018; (6)(6): 1-6 